\title{
Random vibration analysis with radial basis function neural networks
}

\author{
Xi Wang ${ }^{1} \cdot$ Jun Jiang ${ }^{1} \cdot$ Ling Hong $^{1} \cdot$ Jian-Qiao Sun $^{2}$ D \\ Received: 8 October 2021 / Accepted: 1 November 2021 / Published online: 6 December 2021 \\ (c) The Author(s) 2021
}

\begin{abstract}
Random vibrations occur in many engineering systems including buildings subject to earthquake excitation, vehicles traveling on a rough road and off-shore platform in random waves. Analysis of random vibrations for linear systems has been well studied. For nonlinear systems, particularly for multi-degree-of-freedom systems, however, there are still many challenges including analyzing the probability distribution of transient responses of the system. Monte Carlo simulation was considered the only viable method for this task. In this paper, We propose a method to construct semi-analytical transient solutions of the probability distribution of transient responses of nonlinear systems by using the radial basis function neural networks. The activation functions consist of normalized Gaussian probability density functions. Two examples are presented to show the effectiveness of the proposed solution method. The transient probability distributions and response moments of these examples are presented, which have not been reported in the literature before.
\end{abstract}

Keywords Probability density function $\cdot$ Response moment $\cdot$ Nonlinear dynamic system $\cdot$ FPK equation $\cdot$ Neural networks solution

\section{Introduction}

Random vibrations are common in engineering systems. When a system is exposed to random loadings such as airflow, ocean waves and earthquakes, random vibrations occur. Random vibration analysis is an important part of structural and mechanical system design and reliability assessment. Various statistics of the system response such as moments, power spectrum, and probability distribution are often needed for proper design and reliability assessment. Most response statistics can be obtained with the help of the time-varying

Jian-Qiao Sun

jqsun@ucmerced.edu

Xi Wang

767898684@qq.com

Jun Jiang

jun.jiang@mail.xjtu.edu.cn

Ling Hong

hongling@mail.xjtu.edu.cn

1 State Key Laboratory for Strength and Vibration, Xi' an Jiaotong University, Xi' an 710049, People's Republic of China

2 Department of Mechanical Engineering, School of Engineering, University of California, Merced, Merced, CA 95343, USA probability distribution of the response. However, it is a challenge to obtain analytical solutions of the time-varying probability distribution, particularly when the system is nonlinear. This paper presents a radial basis function neural networks (RBF-NN) solution method for computing the time-varying probability density function (PDF) for linear and nonlinear dynamic systems. We further demonstrate the utility of the time-varying PDF for obtaining various response statistics such as response moments.

The probability density function is governed by the wellknown Fokker-Planck-Kolmogorov (FPK) equation. Except for linear dynamic systems, analytical time-varying solutions of the FPK equation have not been reported in the literature [1]. There has been much effort made to obtain exact and approximate steady-state solutions of the PDF [2-6]. Many methods have been developed for steady-state solutions of the PDF including detailed balance [6-8], exponential polynomial closure (EPC) $[9,10]$, stochastic averaging and equivalent linearization $[11,12]$, random walk $[13,14]$, path integral [15-18], finite element method [19,20], finite difference method [21,22] and generalized cell mapping [23]. A highly efficient method for constructing analytical solutions of the steady-state FPK equation for nonlinear stochastic dynamic systems is reported in [24-28].

Neural networks methods for solving partial differential equations have become a popular topic $[29,30]$. The well- 
known classical Ritz method is also implemented with the help of neural networks [31]. The finite element solutions are obtained with the help of neural networks [32]. In the present paper, a radial basis function neural networks solution of the FPK equation is proposed. The activation functions consist of Gaussian probability density functions with the means distributed in the state space. This neural networks approximate solution provides a very efficient and accurate way of computing the time-varying probability density function of nonlinear dynamic systems subject to random excitations. There have been many studies of radial basis functions for regression applications and for solving partial differential equations [33]. The authors of [34] conclude that the radialbasis-function networks having one hidden layer and the same smoothing factor in each kernel are broad enough for universal approximation. An excellent review on radial basis function networks for regression and classification can be found in [35]. This paper will study two challenging examples of nonlinear stochastic dynamic systems to validate the proposed solution method. In particular, we shall report the highly accurate time-varying probability distribution and moments of the response of nonlinear stochastic dynamic systems for the first time in the literature.

The rest of the paper is outlined as follows. Section 2 reviews the FPK equation. Section 3 presents the radial basis function neural networks (RBF-NN) solution of the FPK equation. Section 5 presents random vibration analysis of two challenging examples of nonlinear dynamic systems. The accuracy and computational efficiency of the RBF-NN solution method are investigated and compared with Monte Carlo simulations. The method of Gaussian closure is also considered in the comparison studies. Section 6 concludes the paper.

\section{Problem statement}

Consider random vibrations of an $n$ degree of freedom nonlinear system subject to Gaussian white noise excitations. By introducing the state variables, the equation of motion can be written in a $2 n$-dimensional state space. The resulting equations known as the Itô stochastic differential equation are given by,

$$
\begin{gathered}
d X_{j}(t)=m_{j}(\mathbf{X}, t) d t+\sum_{k=1}^{m} \sigma_{j k}(\mathbf{X}, t) d B_{k}(t), \\
j=1,2, \ldots, 2 n
\end{gathered}
$$

where $X_{j}(t)$ is the $j$ th state variable, and $\left\{B_{k}(t)\right\}$ is an $m$ dimensional vector of independent unit Brownian motions.
The conditional probability density function $p\left(\mathbf{x}, t \mid \mathbf{x}_{0}, t_{0}\right)$ of the system response satisfies the FPK equation

$$
\begin{aligned}
\frac{\partial}{\partial t} p\left(\mathbf{x}, t \mid \mathbf{x}_{0}, t_{0}\right)= & -\sum_{k=1}^{2 n} \frac{\partial}{\partial x_{k}}\left[m_{k}(\mathbf{x}, t) p\right] \\
& +\sum_{j=1}^{2 n} \sum_{k=1}^{2 n} \frac{\partial^{2}}{\partial x_{j} \partial x_{k}}\left[\frac{b_{j k}(\mathbf{x}, t)}{2 !} p\right] \\
= & \mathcal{L}_{F P K}\left[p\left(\mathbf{x}, t \mid \mathbf{x}_{0}, t_{0}\right)\right],
\end{aligned}
$$

subject to the initial condition

$p\left(\mathbf{x}, t_{0} \mid \mathbf{x}_{0}, t_{0}\right)=p\left(\mathbf{x}, t_{0}\right)$

where $\mathcal{L}_{F P K}[\cdot]$ stands for the differential operator of the FPK equation, $p\left(\mathbf{x}, t_{0}\right)$ is a given probability density function, and $b_{j k}=\sum_{l=1}^{m} \sigma_{k l} \sigma_{j l} . m_{k}(\mathbf{x}, t)$ and $b_{j k}(\mathbf{x}, t)$ are known as the drift and diffusion terms. Very often, the initial given is given as a delta function $p\left(\mathbf{x}, t_{0}\right)=\delta\left(\mathbf{x}-\mathbf{x}_{0}\right)$, i.e. the system starts at a point $\mathbf{x}_{0}$ with probability one.

To fully determine the probability density function $p(\mathbf{x}$, $\left.t \mid \mathbf{x}_{0}, t_{0}\right)$ from the FPK equation, we need to specify an initial condition, and proper boundary conditions in the state space. In this work, we shall not consider boundary conditions. Hence, $\mathbf{x} \in \mathbb{R}^{2 n}$.

\section{RBF neural networks solution}

We first present a trial solution for the FPK equation. In particular, we propose a RBF-NN solution with Gaussian activation functions. For convenience, we denote the solution of the FPK equation $p\left(\mathbf{x}, t \mid \mathbf{x}_{0}, t_{0}\right)$ as $p(\mathbf{x}, t)$ subject to the initial condition $p\left(\mathbf{x}, t_{0}\right)$.

\subsection{Trial solution}

Let $\bar{p}(\mathbf{x}, \mathbf{w}(k))$ denote the solution $p(\mathbf{x}, t)$ at time step $k$ where $\mathbf{w}(k)$ are a set of time-varying undetermined parameters. The solution $\bar{p}(\mathbf{x}, \mathbf{w}(k))$ consists of the RBF-NN with Gaussian activation functions given by,

$\bar{p}(\mathbf{x}, \mathbf{w}(k))=\sum_{j=1}^{N_{G}} w_{j}(k) g\left(\mathbf{x}, \boldsymbol{\mu}_{j}, \boldsymbol{\Sigma}_{j}\right)$

where $N_{G}$ is the number of Gaussian functions, $\mathbf{x} \in \mathbb{R}^{2 n}$ is the position vector. The model parameters $w_{j}$ form a vector $\mathbf{w}(k)=\left[w_{1}(k), w_{2}(k), \ldots, w_{N_{G}}(k)\right]^{T}$. The time depen- 
dence of the assumed solution $\bar{p}$ is through that of the coefficients $\mathbf{w}(k)$. The index $k$ indicates the time step such that $t=t_{0}+k \tau, k=0,1,2, \ldots$.

$g\left(\mathbf{x}, \boldsymbol{\mu}_{j}, \boldsymbol{\Sigma}_{j}\right)$ is a neuron in the form of a Gaussian function with the mean $\boldsymbol{\mu}_{j}$ and covariance matrix $\boldsymbol{\Sigma}_{j}$.

$$
\begin{aligned}
g\left(\mathbf{x}, \boldsymbol{\mu}_{j}, \boldsymbol{\Sigma}_{j}\right)= & \frac{1}{\sqrt{(2 \pi)^{2 n} \operatorname{det}\left(\boldsymbol{\Sigma}_{j}\right)}} \exp \left[-\frac{1}{2}\left(\mathbf{x}-\boldsymbol{\mu}_{j}\right)^{T} \mathbf{\Sigma}_{j}^{-1}\right. \\
& \left.\cdot\left(\mathbf{x}-\boldsymbol{\mu}_{j}\right)\right] .
\end{aligned}
$$

We should point out that the radial basis Gaussian function in the literature refers to the special case when $\boldsymbol{\Sigma}_{j}=\sigma_{j}^{2} \mathbf{I}$, $\operatorname{det}\left(\boldsymbol{\Sigma}_{j}\right)=\left(\sigma_{j}^{2}\right)^{2 n}, \boldsymbol{\Sigma}_{j}^{-1}=\sigma_{j}^{-2} \mathbf{I}$ and

$$
\begin{aligned}
g\left(\mathbf{x}, \boldsymbol{\mu}_{j}, \boldsymbol{\Sigma}_{j}\right) & =\frac{1}{\sqrt{(2 \pi)^{2 n}\left(\sigma_{j}^{2}\right)^{2 n}}} \exp \left[-\frac{1}{2 \sigma_{j}^{2}}\left\|\mathbf{x}-\boldsymbol{\mu}_{j}\right\|^{2}\right] \\
& =\prod_{i=1}^{2 n} \frac{1}{\sqrt{2 \pi} \sigma_{j}} \exp \left[-\frac{1}{2 \sigma_{j}^{2}}\left(x_{i}-\mu_{j, i}\right)^{2}\right],
\end{aligned}
$$

where $\mathbf{I}$ is an identity matrix, and $\|\cdot\|$ denote the norm of a vector, and $\mu_{j, i}$ is the $i$ th component of the mean vector $\boldsymbol{\mu}_{j}$. The radial form of the Gaussian functions makes computations a lot easier and is used in the numerical studied reported later.

It is noted that the transient probability density function depends on the initial condition. When the initial probability distribution is highly concentrated such as a delta function $\delta\left(\mathbf{x}-\mathbf{x}_{0}\right)$, we can use the short-time analytical approximate solution to compute the probability density over one or a few time steps $\tau$ to avoid the need for extremely small meshes.

The RBF-NN PDF solution must satisfy the normalization condition,

$$
\int_{\mathbb{R}^{2 n}} \bar{p}(\mathbf{x}, \mathbf{w}(k)) d \mathbf{x}=1, \text { for } k \geq 0
$$

Because of the proper definition of the Gaussian activation functions such that

$\int_{\mathbb{R}^{2 n}} g\left(\mathbf{x}, \boldsymbol{\mu}_{j}, \boldsymbol{\Sigma}_{j}\right) d \mathbf{x}=1$, for all $j$

the normalization condition for the RBF-NN PDF solution becomes

$\sum_{j=1}^{N_{G}} w_{j}(k)=1$, for $k \geq 0$.

Recall the initial condition $p_{0}(\mathbf{x})$ of the FPK equation (3). We apply the solution given in Eq. (4) with a proper choice of the mean $\boldsymbol{\mu}_{j}$ and covariance matrix $\boldsymbol{\Sigma}_{j}$. We obtain the initial coefficients $\mathbf{w}(0)$. Let $\tau$ be a small time step for integrating the FPK equation forward in time, and $\mathbf{w}(k)$ be the coefficient vector at time $t=k \tau, k=0,1,2, \ldots$.

We apply the finite difference to compute the time derivative

$\frac{\partial \bar{p}}{\partial t}=\frac{1}{\tau}[\bar{p}(\mathbf{x}, \mathbf{w}(k+1))-\bar{p}(\mathbf{x}, \mathbf{w}(k))]+\epsilon_{t}$,

where $\epsilon_{t}$ is the truncation error of the first order finite difference approximation.

Substitute the solution in Eq. (4) and the time derivative in Eq. (10) to Eq. (2). We define a local error of the equation as

$$
\begin{aligned}
e(\mathbf{x}, \mathbf{w}(k)) & =-\bar{p}(\mathbf{x}, \mathbf{w}(k))+\bar{p}(\mathbf{x}, \mathbf{w}(k-1))+\mathcal{L}_{F P K}[\bar{p}(\mathbf{x}, \mathbf{w}(k))] \tau \\
& =\bar{p}(\mathbf{x}, \mathbf{w}(k-1))+\sum_{j=1}^{N_{G}} s_{j}(\mathbf{x}) w_{j}(k), \text { for } k=1,2, \ldots
\end{aligned}
$$

where

$s_{j}(\mathbf{x})=-g\left(\mathbf{x}, \boldsymbol{\mu}_{j}, \boldsymbol{\Sigma}_{j}\right)+\tau \mathcal{L}_{F P K}\left[g\left(\mathbf{x}, \boldsymbol{\mu}_{j}, \boldsymbol{\Sigma}_{j}\right)\right]$.

The explicit expression for $s_{j}(\mathbf{x})$ after all the differentiations are carried out can be obtained symbolically in order to speed up the computation. Note that at step $k, \bar{p}(\mathbf{x}, \mathbf{w}(k-1))$ is fully determined. The truncation error $\epsilon_{t}$ can be thought of as being absorbed in the error $e(\mathbf{x}, \mathbf{w}(k))$.

We define an integrated squared error together with a Lagrange multiplier $\lambda(k)$ for the normalization condition in Eq. (9) as

$J(\mathbf{w}(k), \lambda(k))=\int_{\mathbb{R}^{2 n}} \frac{1}{2} e^{2}(\mathbf{x}, \mathbf{w}(k)) d \mathbf{x}+\lambda(k)\left(\sum_{j=1}^{N_{G}} w_{j}(k)-1\right)$.

We should point out that this step of optimization is important to correct the truncation error so that at every integration time step, the solution is obtained in the sense of the least squared error.

We select $\mathbf{w}(k)$ and $\lambda(k)$ to minimize $J$. Define a parameter vector as

$\mathbf{c}(k)=\left[w_{1}(k), w_{2}(k), \ldots, w_{N_{G}}(k), \lambda(k)\right]^{T}=\left[\mathbf{w}^{T}(k), \lambda(k)\right]^{T}$.

For convenience, we shall denote $J(\mathbf{w}(k), \lambda(k))$ as $J(\mathbf{c}(k))$. We shall derive necessary conditions for minimization of $J(\mathbf{c}(k))$. 


\subsection{Motivation for choosing Gaussian functions}

It is well known that the generalized cell mapping (GCM) with the short-time Gaussian approximation (STGA) is a highly effective method for computing the probabilistic response of nonlinear stochastic systems [1]. In the GCMSTGA method, the one-step transition probability matrix of nonlinear stochastic systems starting from each initial cell has a Gaussian distribution over a short time. The probabilistic response of the stochastic system is fully determined by the one-step transition probability matrix from all the initial cells in a given domain. The GCM-STGA method indicates that at every mapping step, the probabilistic distribution of the response is a linear combination of many Gaussian probability distributions. This is consistent with the RBF-NN solution given in Eq. (4).

\subsection{Sampling method for integration}

Note that the computation of $J(\mathbf{c}(k))$ involving integrations in high dimensional space can be intensive. Instead of integration, we can randomly sample a large number of points $\mathbf{x}_{i} \in D$ where $D \subset \mathbb{R}^{2 n}$ is a large enough domain in order to calculate an approximate value of the integrated squared error as

$J_{S}(\mathbf{c}(k))=\sum_{i=1}^{N_{s}} \frac{1}{2} e^{2}\left(\mathbf{x}_{i}, \mathbf{w}(k)\right)+\lambda(k)\left(\sum_{j=1}^{N_{G}} w_{j}(k)-1\right), \mathbf{x}_{i} \in D$

where $N_{s}$ is the number of sampled points. The sampled performance index $J_{S}(\mathbf{c})$ can be written in the following form.

$$
\begin{aligned}
J_{s}(\mathbf{c}(k))= & \sum_{i=1}^{N_{s}} \frac{1}{2}\left(\sum_{j=1}^{N_{G}} \sum_{l=1}^{N_{G}} s_{j}\left(\mathbf{x}_{i}\right) s_{l}\left(\mathbf{x}_{i}\right) w_{j}(k) w_{l}(k)\right) \\
& +\sum_{i=1}^{N_{s}} \sum_{j=1}^{N_{G}} s_{j}\left(\mathbf{x}_{i}\right) \bar{p}\left(\mathbf{x}_{i}, \mathbf{w}(k-1)\right) w_{j}(k) \\
& +\frac{1}{2} \sum_{i=1}^{N_{s}} \bar{p}^{2}\left(\mathbf{x}_{i}, \mathbf{w}(k-1)\right)+\lambda(k)\left(\sum_{j=1}^{N_{G}} w_{j}(k)-1\right) \\
& \equiv \mathbf{c}^{T} \mathbf{A c}(k)+\mathbf{c}^{T}\left[\mathbf{b}_{p}(k)-\mathbf{b}_{\lambda}\right]+d(k-1),
\end{aligned}
$$

where the matrix $\mathbf{A} \in \mathbb{R}^{\left(N_{G}+1\right) \times\left(N_{G}+1\right)}$ is defined with the help of a data matrix $\mathbf{S} \in \mathbb{R}^{N_{G} \times N_{s}}$. The vectors $\mathbf{b}_{\lambda}$ and $\mathbf{b}_{p} \in$ $\mathbb{R}^{\left(N_{G}+1\right) \times 1}$ together with a scalar $d(k-1)$ are also defined below.

$$
\begin{aligned}
\mathbf{S} & =\left[S_{j l}\right]=\left[s_{j}\left(\mathbf{x}_{l}\right)\right], \\
\mathbf{G} & =\left[G_{j l}\right]=\left[g_{j}\left(\mathbf{x}_{l}\right)\right], \\
\mathbf{b}_{\lambda} & =[0,0, \ldots, 0,1]^{T},
\end{aligned}
$$

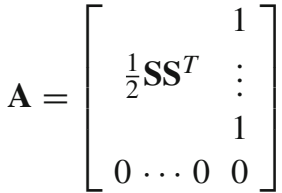

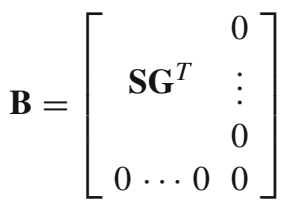

and

$$
\begin{aligned}
b_{p j}(k) & =\sum_{i=1}^{N_{s}} s_{j}\left(\mathbf{x}_{i}\right) \bar{p}\left(\mathbf{x}_{i}, \mathbf{w}(k-1)\right) \\
& =\sum_{i=1}^{N_{s}} s_{j}\left(\mathbf{x}_{i}\right) \sum_{l=1}^{N_{G}} w_{l}(k-1) G\left(\mathbf{x}_{i}, \boldsymbol{\mu}_{l}, \boldsymbol{\Sigma}_{l}\right), \\
\mathbf{b}_{p}(k) & =\left[b_{p 1}, b_{p 2}, \ldots, b_{p N_{G}}, 0\right]^{T}=\mathbf{B c}(k-1), \\
d(k-1) & =\sum_{i=1}^{N_{s}} \bar{p}^{2}\left(\mathbf{x}_{i}, \mathbf{w}(k-1)\right) .
\end{aligned}
$$

The necessary condition for minimizing $J_{S}$ with respect to the vector $\mathbf{c}$ reads

$$
\frac{\partial J}{\partial \mathbf{c}(k)}=\left(\mathbf{A}+\mathbf{A}^{T}\right) \mathbf{c}(k)+\mathbf{b}_{p}(k)-\mathbf{b}_{\lambda}=0 .
$$

The optimal coefficient vector is given by

$\mathbf{c}_{\text {opt }}(k)=\left(\mathbf{A}+\mathbf{A}^{T}\right)^{-1}\left[\mathbf{b}_{\lambda}-\mathbf{B c}(k-1)\right]$,

when the inverse of the matrix $\left(\mathbf{A}+\mathbf{A}^{T}\right)$ exists. The existence of the inversion heavily depends on the so-called data matrix S. As is well-known in the regression research [36], the data matrix also determines the overall performance of the RBFNN PDF solution in Eq. (4).

\section{Response moments}

With the probability density function on hand, many response statistics can be computed including the moments. The first and second order moments of the system response at the $k$ th time step can be computed as follows.

$$
\begin{aligned}
E[\mathbf{X}](k) & =\int_{\mathbb{R}^{2 n}} \mathbf{x} \bar{p}(\mathbf{x}, \mathbf{w}(k)) d \mathbf{x} \\
& =\sum_{j=1}^{N_{G}} w_{j}(k) \int_{\mathbb{R}^{2 n}} \mathbf{x} g\left(\mathbf{x}, \boldsymbol{\mu}_{j}, \boldsymbol{\Sigma}_{j}\right) d \mathbf{x} \\
& =\sum_{j=1}^{N_{G}} w_{j}(k) \boldsymbol{\mu}_{j},
\end{aligned}
$$


The expectation with respect to the $j$ th Gaussian function has the following property.

$$
\begin{aligned}
E\left[\mathbf{X}^{T} \mathbf{X}\right]= & E\left[\left(\mathbf{X}-\boldsymbol{\mu}_{j}\right)^{T}\left(\mathbf{X}-\boldsymbol{\mu}_{j}\right)\right] \\
& +E\left[\left(\mathbf{X}-\boldsymbol{\mu}_{j}\right)^{T}\right] \boldsymbol{\mu}_{j}+\boldsymbol{\mu}_{j} E\left[\left(\mathbf{X}-\boldsymbol{\mu}_{j}\right)^{T}\right]+\boldsymbol{\mu}_{j}^{T} \boldsymbol{\mu}_{j} .
\end{aligned}
$$

By definition, we have

$$
\begin{aligned}
& E\left[\left(\mathbf{X}-\boldsymbol{\mu}_{j}\right)^{T}\left(\mathbf{X}-\boldsymbol{\mu}_{j}\right)\right]=\boldsymbol{\Sigma}_{j} \\
& E\left[\left(\mathbf{X}-\boldsymbol{\mu}_{j}\right)\right]=0
\end{aligned}
$$

Hence, we have the correlation matrix, i.e. the second order moments, as

$$
\begin{aligned}
E\left[\mathbf{X}^{T} \mathbf{X}\right](k) & =\int_{\mathbb{R}^{2 n}} \mathbf{x}^{T} \mathbf{x} \bar{p}(\mathbf{x}, \mathbf{w}(k)) d \mathbf{x} \\
& =\sum_{j=1}^{N_{G}} w_{j}(k)\left[\boldsymbol{\Sigma}_{j}+\boldsymbol{\mu}_{j}^{T} \boldsymbol{\mu}_{j}\right],
\end{aligned}
$$

Once the first and second moments are obtained, we can compute the response moments of any order. Let us use a typical moment $E\left[X_{i}^{p} X_{k}^{q}\right]$ to illustrate the computational process by using the radial basis Gaussian functions in Eq. (6). Because of the special radial form of the Gaussian functions, it implies that for the $j$ th Gaussian distribution $g\left(\mathbf{x}, \boldsymbol{\mu}_{j}, \boldsymbol{\Sigma}_{j}\right)$, the random variables $X_{i}$ and $X_{k}$ can be viewed as being independent for $i \neq k$. Hence, the expectation with respect to this Gaussian distribution can be written as

$$
E\left[X_{i}^{p} X_{k}^{q}\right]=E\left[X_{i}^{p}\right] \cdot E\left[X_{k}^{q}\right]
$$

That is, we only need to focus on the computation of the moment $E\left[X_{i}^{p}\right]$ with respect to the $j$ th Gaussian distribution. To compute this quantity, we convert it to the central moment as

$$
E\left[\left(X_{i}-\mu_{j, i}+\mu_{j, i}\right)^{p}\right]=\sum_{l=0}^{p} C_{l}^{p} E\left[\left(X_{i}-\mu_{j, i}\right)^{p-l}\right] \mu_{j, i}^{l}
$$

where

$C_{l}^{p}=\frac{p !}{(p-l) ! l !}$

The central moments determined by the $j$ th Gaussian function $g\left(\mathbf{x}, \boldsymbol{\mu}_{j}, \boldsymbol{\Sigma}_{j}\right)$ are given by

$E\left[\left(X_{i}-\mu_{j, i}\right)^{p}\right]= \begin{cases}0 & \text { if } p \text { is odd, } \\ \sigma_{j}^{p}(p-1) ! & \text { if } p \text { is even. }\end{cases}$

With the help of this result, arbitrary cross moments such as $E\left[X_{i}^{p} X_{k}^{q}\right]$ can be expressed in terms of lower order moments with respect to each Gaussian function.

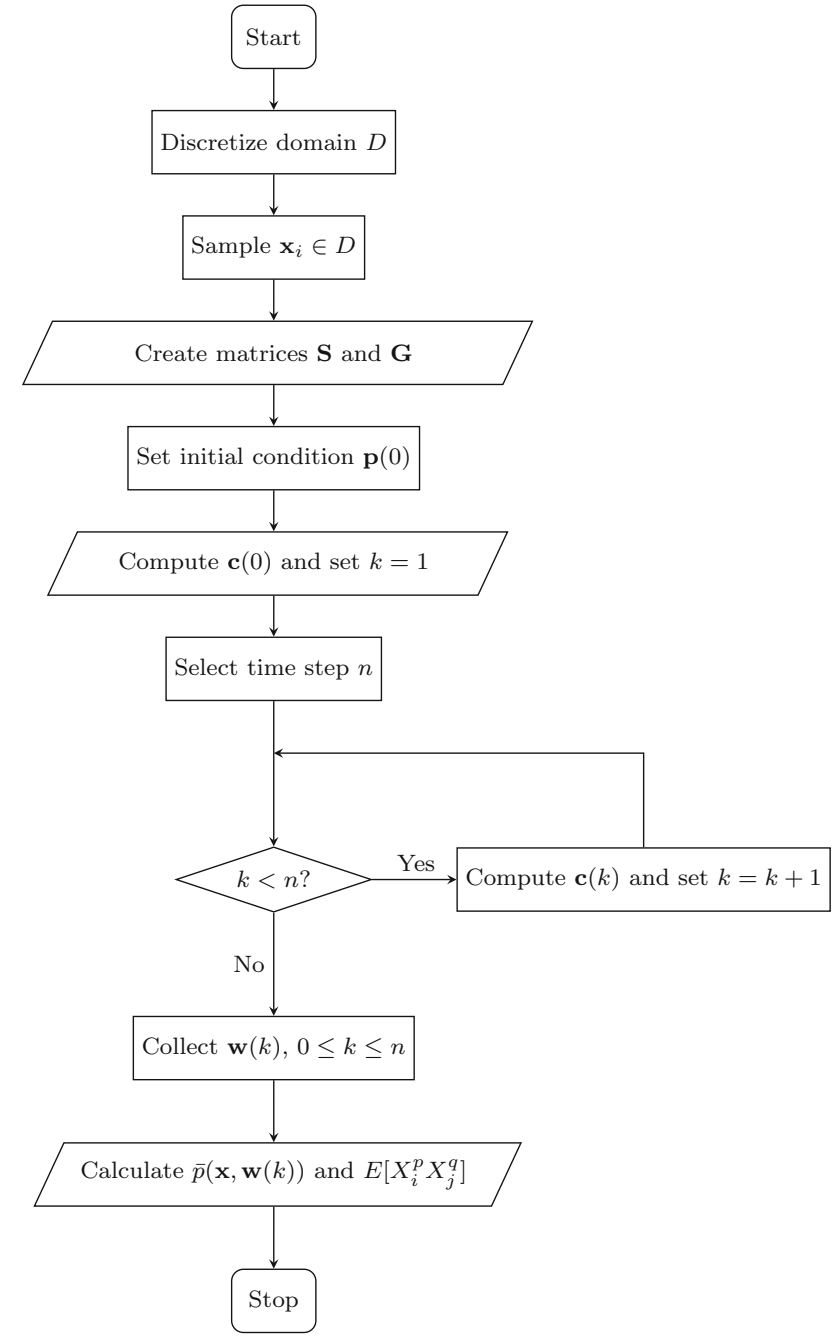

Fig. 1 The flowchart of the RBF-NN method

This demonstrates another advantage of choosing the true probability density as the basis function. The response moments of any order can be computed without numerical integration in the space $\mathbb{R}^{2 n}$.

The logic of the proposed RBF-NN method for the path integral solution of the FPK equation is summarized in the following flowchart (Fig. 1).

\section{Numerical examples}

In this section, we present two numerical examples of vibration analysis to illustrate the application of the proposed RBF-NN PDF solution method. As discussed in [34], the radial-basis-function networks with one hidden layer and the same smoothing factor have the property of universal approximation, meaning that the error of the solution can be sufficiently small when enough terms of the RBF-NN solution are used. In an early study, we have demonstrated the 
accuracy of the PDF solution. In this paper, we shall not directly address this issue. Instead, we apply the RBF-NN PDF solution to compute transient PDF solution and response moments of the system, and investigate the accuracy of the RBF-NN solutions with the help of Monte Carlo simulations.

\section{Example one}

Consider a first order nonlinear system

$\frac{d X}{d t}=a X-b X^{3}+W_{1}(t)$

where $b>0$, and $W_{1}(t)$ denotes the Gaussian white noise excitation with intensity $2 D_{1}$. The drift and diffusion coefficients of the FPK equation are given by,

$m_{1}(x, t)=a x-b x^{3}, \quad b_{22}=2 D_{1}$.

We choose the computational domain in the state space to be $D=[-5,5]$ and the parameters $a=1, b=2$ and $D_{1}=3$. The number of Gaussian functions is $N_{G}=101$. The means of all the Gaussian functions are at the grid points, while the standard deviations of all the Gaussian functions are taken to be the same constant $\sigma_{j}=h$ where $h$ is the grid size $h=10 / 101$. The number of sampling point $N_{s}=2 N_{G}$, which was found to be sufficient. We should point out that when $a>0$, the steady-state PDF function of the system is bi-modal because $x=0$ is an unstable equilibrium.

Consider an initial condition as $x_{0}=0$ at $t=0$ with probability one. We choose a small time step for integration as $\tau=0.01$, and construct a short-time Gaussian approximation of the PDF with the mean $x_{0}+m\left(x_{0}, 0\right) \tau=0$ and variance $2 b\left(x_{0}, 0\right) \tau=0.12$.

Figure 2 presents evolutions of the response PDF and a comparison of the RBF-NN PDF solution with Monte Carlo simulations. In this example, we generate $10^{5}$ sample time histories in simulations in order to compute all the response statistics reported herein. Simulations take $103 \mathrm{~s}$. As a comparison, the RBF-NN method takes $2.83 \mathrm{~s}$ to compute the same set of solutions. The overall rms error of the RBF-NN PDF solution compared to Monte Carlo simulation is 0.0141 . The agreement between the two results is indeed excellent.

Let $m_{p}=E\left[X^{p}\right]$ denote the $p$ th order moment of the response. We apply Eqs. (29) and (32) to compute the first and second order moments of the response. Furthermore, we shall compare the moments obtained by the RBF-NN PDF solution with those by Monte Carlo simulations and Gaussian closure approximation.

The Gaussian closure makes use of the following relationships of higher order moments to the lower order ones.

$m_{3}=3 m_{2} m_{1}-2 m_{1}^{3}$,
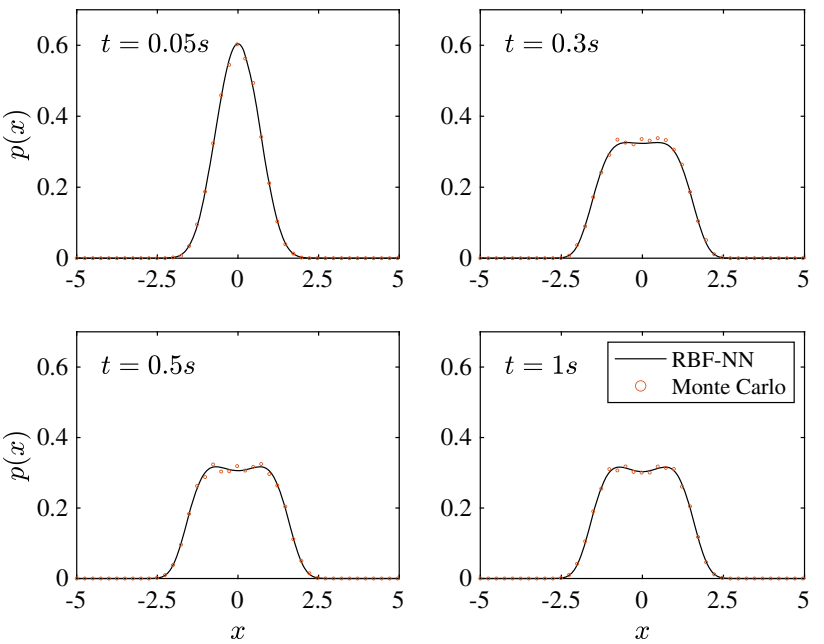

Fig. 2 The evolution of the PDF of system (37) obtained by the proposed method and Monte Carlo simulation

$m_{4}=3 m_{2}^{2}-2 m_{1}^{4}$,

The equations for the first and second order moments of system (37) obtained with the Gaussian closure are given by

$\frac{d m_{1}}{d t}=a m_{1}-b\left(3 m_{2} m_{1}-2 m_{1}^{3}\right)$,

$\frac{d m_{2}}{d t}=2 a m_{2}-6 b m_{2}^{2}+4 b m_{1}^{4}+2 D$,

We first consider a case when $a=1>0$ and the steadystate PDF function of the system is bi-modal. The bi-modal distribution is certainly far away from being Gaussian. Figure 3 shows the time histories of the first and second order moments of the system response by the the RBF-NN solution, the Gaussian closure method and Monte Carlo simulations. It is seen from the figure that the response moments obtained with the PDF of the RBF-NN solution is highly accurate as compared to Monte Carlo simulations, while the Gaussian closure performs poorly because the underlining process is far away from being Gaussian.

The Gaussian closure method is only good for weakly nonlinear system when the system response distribution is nearly Gaussian. When the parameter $a<0$, the response distribution of system (37) is nearly Gaussian with a single peak at $x=0$. Let $a=-1, b=0.1$ and $D_{1}=3$. Figure 4 shows the first and second order response moments of the system by the the RBF-NN solution, the Gaussian closure method and Monte Carlo simulations. The performance of the Gaussian closure method improves significantly because the response of the system is nearly Gaussian.

Figure 5 shows the steady-state second order moments of the system response as a function of the parameter $b$. It should be noted that when $b$ is large, even when $a>0$, the two-peaks of the probability distribution move closer to each 

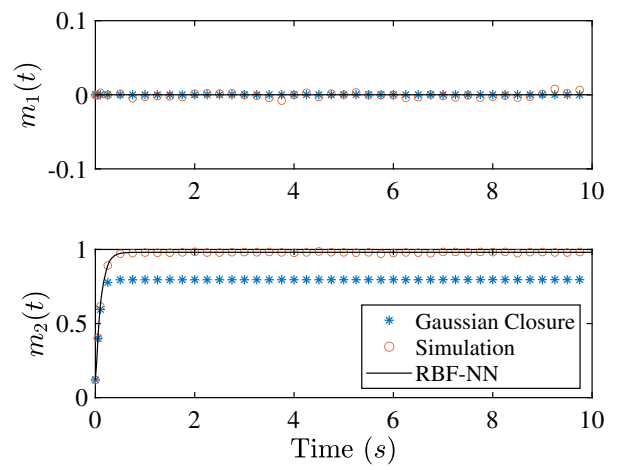

Fig. 3 The first and second order moments of the system response by the proposed method, Gaussian closure and Monte Carlo simulations. $a=1$
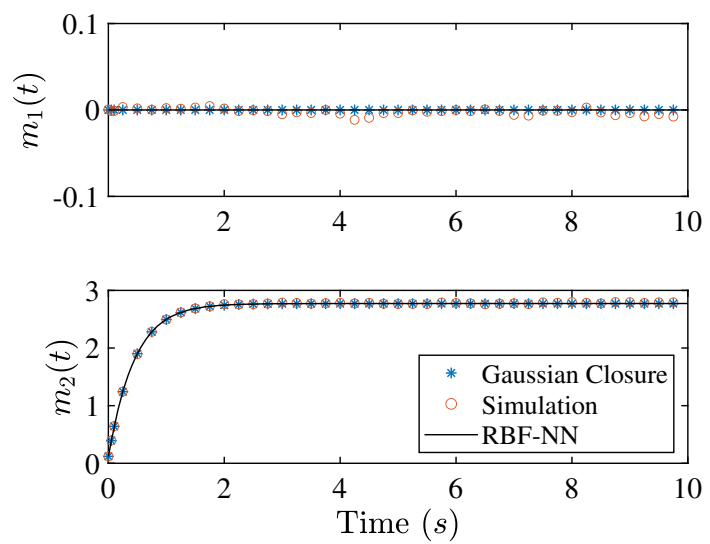

Fig. 4 The first and second order moments of the system response by the proposed method, Gaussian closure and Monte Carlo simulations. $a=-1$

other, resulting a distribution which looks more like a Gaussian function. Hence, as $b$ increases, the error of the Gaussian closure solution decreases. The RBF-NN solution is consistently accurate as compared to Monte Carlo simulations for all the parameters we considered.

In Fig. 5 for $a=1$, the average error in $m_{2}$ over the range of parameter $b$ of the RBF-NN solution is $0.2580 \%$, while the same error of the Gaussian closure solution is $24.7659 \%$, which is about 96 times bigger than that of the RBF-NN solution. For $a=-1$, the average error in $m_{2}$ over the range of parameter $b$ of the RBF-NN solution is $0.2948 \%$, while the same error of the Gaussian closure solution is $7.4546 \%$, which is about 25 times bigger than that of the RBF-NN solution. In this case, the response is close to be Gaussian.

\section{Example Two}

Consider a strongly nonlinear Van der Pol oscillator under external Gaussian white noise excitation.

$\frac{d X}{d t}=Y$,
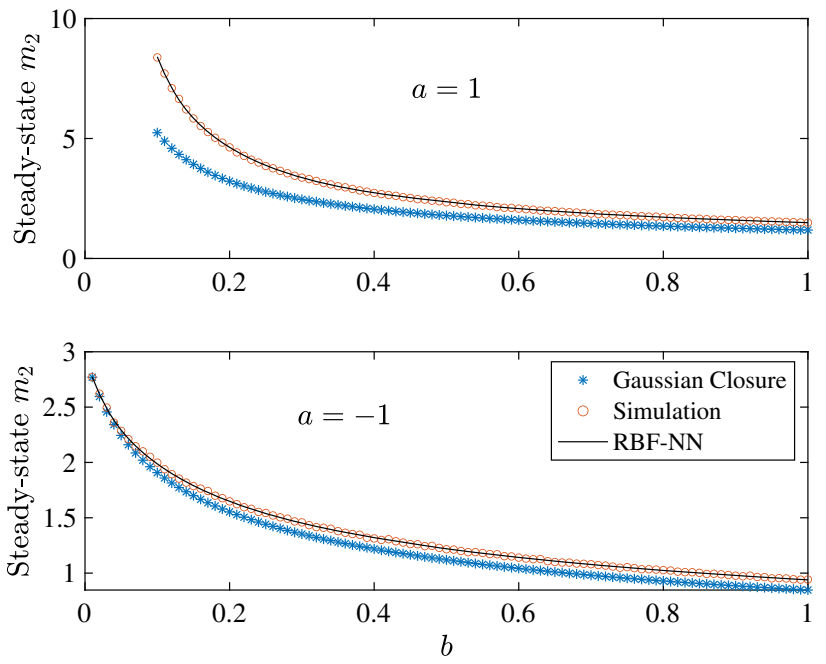

Fig. 5 The steady-state second order moments of the system response as a function of the parameter $b$ by the proposed method, Gaussian closure and Monte Carlo simulations

$$
\frac{d Y}{d t}=-\beta\left(X^{2}-1\right) Y-X+W_{1}(t)
$$

where $\beta=1.5$ is constant. $W_{1}(t)$ denotes the Gaussian white noise excitation with intensity $2 D_{1}=0.5$. This system has long been a challenging example in the study of stochastic dynamic systems. There has been no report in the literature on the transient response of the PDF. Here, we apply the RBF-NN solution method to obtain the transient probability distribution of the system response and the time-varying moments.

Let us take the domain of interest in the state space as $D_{G}=[-3,3] \times[-5.5,5.5]$ and partition it into a $N_{G} \times N_{G}$ grid where $N_{G}=100$. At each grid, there is a Gaussian function. Every Gaussian function is assumed to have the same standard deviation $\sigma_{j}=h$ where $h$ is the grid size. We have found from computational exercises that it is beneficial to sample points for computing the data matrix in a slightly larger domain than $D_{G}$. In this example, we take $D_{s}=[-4,4] \times[-7,7]$ and uniformly sample $N_{s} \times N_{s}$ points in $D_{s}$ where $N_{s}=200$. We use the short-time Gaussian approximation of the PDF to deal with the initial condition with probability one at $(x, y)=(0,0)$ when $t=0$. When $t=\tau$, the response distribution is approximately Gaussian with the mean $[0,0]$ and variance $[0.05,0.05]$, according to the short-time Gaussian approximation.

Figure 6 compares the steady-state PDFs of the Van der Pol oscillator obtained by the RBF-NN solution method and Monte Carlo simulations. $10^{6}$ samples are generated in simulations, which takes $1555.3 \mathrm{~s}$. As a comparison, the RBF-NN method takes $2226.6 \mathrm{~s}$ when $N_{G}=100$ and $N_{s}=200$, and $540 \mathrm{~s}$ when $N_{G}=60$ and $N_{s}=120$. The overall rms error of the RBF-NN PDF solution compared to Monte Carlo 

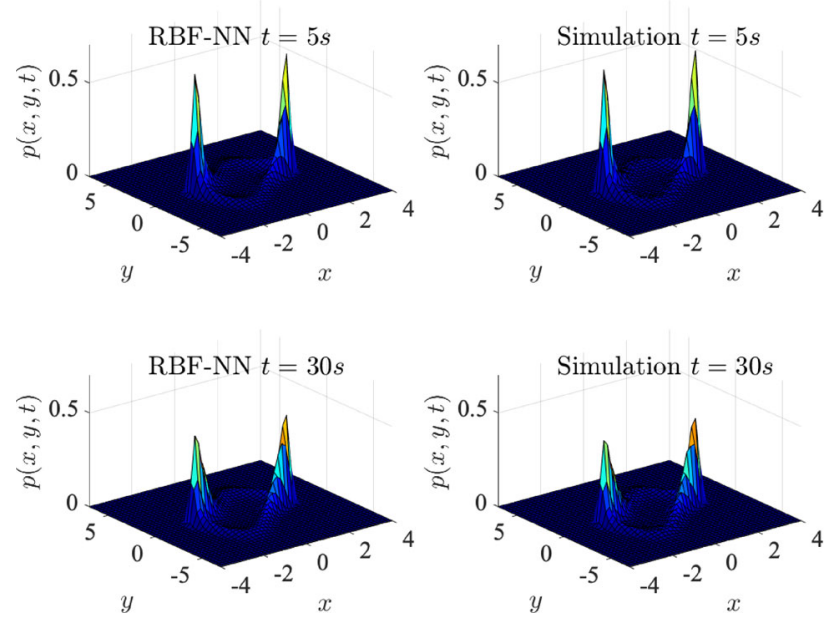

Fig. 6 Comparison of the PDF solution of the Van der Pol oscillator obtained by the proposed method and Monte Carlo simulations

simulation is 0.0196 . We shall further examine the accuracy of the solution by considering the solutions of the response moments.

Let $m_{p q}=E\left[X^{p} Y^{q}\right]$. The equations governing the moment evolution of the Van der Pol oscillator after applying Gaussian closure are obtained as,

$$
\begin{aligned}
& \frac{d m_{10}}{d t}=m_{01}, \\
& \frac{d m_{01}}{d t}=-\beta\left(m_{20}-1\right) m_{01}-m_{10}, \\
& \frac{d m_{20}}{d t}=2 m_{11}, \\
& \frac{d m_{11}}{d t}=-\beta\left(3 m_{20} m_{11}-2 m_{10}^{3} m_{01}\right)+\beta m_{11}-m_{20}+m_{02}, \\
& \frac{d m_{02}}{d t}=-2 \beta m_{20} m_{02}+2 \beta m_{02}-2 m_{11}+2 D_{1} .
\end{aligned}
$$

We shall not show the first order response moments of the system because the solutions obtained by all the methods under consideration are nearly zero as they should be. Figure 7 shows the second order response moments of the system by the the RBF-NN solution, the Gaussian closure method and Monte Carlo simulations. It is seen from the figure that the RBF-NN solution is consistently accurate as compared to Monte Carlo simulations, while $m_{20}$ and $m_{02}$ obtained by the Gaussian closure method have significant steady-state error in addition to poor transient performance. The reason for poor performance of the Gaussian closure is explained by the PDF shapes in Fig. 6. For $t>0$ away from the concentrated initial condition, the PDF of the Van der Pol oscillator is far away from being Gaussian.

However, at any time $t>0$, the geometrically complex PDF of the Van der Pol oscillator can always be expressed as a linear combination of radial basis Gaussian functions given
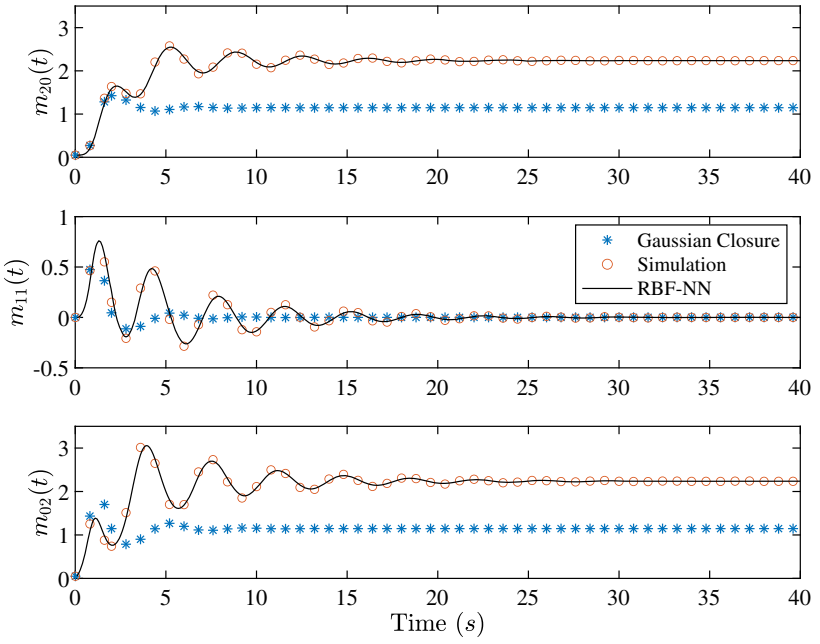

Fig. 7 Comparison of the transient response moments of the Van der Pol oscillator obtained by the proposed method and Monte Carlo simulations

in Eq. (4) with a proper choice of the means and standard deviations. Furthermore, when $N_{G}$ is sufficiently large, the error of the RBF-NN solution converges to zero [34].

Let us know discuss the solution errors of response moments in reference to the simulation results. The steadystate error averaged over the last 100 data points in $m_{20}$ of the RBF-NN solution is 0.00079333 , which represents a very small percentage of the response moment. The same error of the Gaussian closure solution is 1.0908 . This is over $40 \%$ of the response moment $m_{20}$ according to the simulation results. The steady-state error averaged over the last 100 data points in $m_{02}$ of the RBF-NN solution is 0.0018222 , which is also a very small percentage of the response moment. The same error of the Gaussian closure solution is still 1.0908, and is over $40 \%$ of the response moment $m_{02}$. The steady-state error averaged over the last 100 data points in $m_{11}$ is 0.00072965 of the RBF-NN solution, and 0.00080836 of the Gaussian closure solution. The exact steady-state value of $m_{11}$ is known to be zero.

\section{Conclusions}

This paper has presented a radial basis function neural networks approach with Gaussian activation functions for constructing the time-varying probability density function of the FPK equation of nonlinear stochastic dynamic systems with high accuracy and computational efficiency. Such a highly accurate solution of time-varying probability density function for nonlinear stochastic dynamic systems enables us to analyze various response statistics of random vibrations of nonlinear dynamic systems. The response moments are considered as an example of applications. The choice 
of the standard Gaussian probability density functions as activation functions makes computations of other response statistics such as moments highly efficient as illustrated in the paper. The aforementioned features are the pros of the proposed method as compared to other methods such the finite element method, random walk and direct simulations. Since the solution obtained with the help of the proposed method is numerical in nature, compared to the methods such as stochastic averaging and equivalent linearization which deliver approximate solutions in analytical form, it is at a disadvantage.

Two challenging nonlinear dynamic systems are studied with the radial basis function neural networks, including the notorious Van der Pol oscillator, which defies analytical treatments. The time-varying probability density functions as well as response moments of the system are presented which are highly accurate and are computed with short CPU time compared to simulations. It is believed that such accurate time-varying response statistics for nonlinear dynamic systems have not been reported in the literature before.

Acknowledgements This work was supported by the National Natural Science Foundation of China (Grant Numbers 11972274 and 11772243).

Author contributions The authors all contributed to the work in terms of programming (Wang), mathematical formulation (Hong, Jiang and Sun) and manuscript drafting and editing (Sun).

Availability of data and material There is no data associated with this paper.

\section{Declarations}

Conflict of interest There is no conflict of interest between the funding agency and the authors.

Open Access This article is licensed under a Creative Commons Attribution 4.0 International License, which permits use, sharing, adaptation, distribution and reproduction in any medium or format, as long as you give appropriate credit to the original author(s) and the source, provide a link to the Creative Commons licence, and indicate if changes were made. The images or other third party material in this article are included in the article's Creative Commons licence, unless indicated otherwise in a credit line to the material. If material is not included in the article's Creative Commons licence and your intended use is not permitted by statutory regulation or exceeds the permitted use, you will need to obtain permission directly from the copyright holder. To view a copy of this licence, visit http://creativecomm ons.org/licenses/by/4.0/.

\section{References}

1. Sun JQ (2006) Stochastic dynamics and control. Elsevier Science Ltd, Oxford

2. Zhu WQ, Cai GQ, Lin YK (1990) On exact stationary solutions of stochastically perturbed Hamiltonian systems. Probab Eng Mech $5(2): 84-87$
3. Dimentberg MF (1982) An exact solution to a certain non-linear random vibration problem. Int J Non-Linear Mech 17(4):231-236

4. Caughey TK, Ma F (1982) The exact steady-state solution of a class of non-linear stochastic-systems. Int J Non-Linear Mech 17(3):137-142

5. Cai GQ, Lin YK (1988) On exact stationary solutions of equivalent non-linear stochastic-systems. Int J Non-Linear Mech 23(4):315325

6. Lin YK, Cai GQ (2004) Probabilistic structural dynamics: advanced theory and applications. McGraw-Hill, New York

7. Yong Y, Lin YK (1987) Exact stationary-response solution for 2ndorder nonlinear-systems under parametric and external white-noise excitations. J Appl Mech Trans ASME 54(2):414-418

8. Lin YK, Cai GQ (1988) Exact stationary response solution for second order nonlinear systems under parametric and external white noise excitations: Part ii. J Appl Mech Trans ASME 55(3):702-705

9. Er GK (2011) Methodology for the solutions of some reduced Fokker-Planck equations in high dimensions. Ann Phys 523(3):247-258

10. Er GK (2014) Probabilistic solutions of some multi-degree-offreedom nonlinear stochastic dynamical systems excited by filtered Gaussian white noise. Comput Phys Commun 185(4):1217-1222

11. Anh ND, Zakovorotny VL, Hao DN (2014) Response analysis of van der pol oscillator subjected to harmonic and random excitations. Probab Eng Mech 37:51-59

12. Zhu HT, Guo SS (2015) Periodic response of a duffing oscillator under combined harmonic and random excitations. J Vib Acoust Trans ASME 137(4):041015

13. Toland RH, Yang CY, Hsu KC (1972) Non-stationary random vibration of non-linear structures. Int J Non-Linear Mech 7(4):395406

14. Roberts JB (1981) Transient-response of non-linear systems to random-excitation. J Sound Vib 74(1):11-29

15. Wehner MF, Wolfer WG (1983) Numerical evaluation of pathintegral solutions to Fokker-Planck equations. Phys Rev A 27(5):2663-2670

16. Wehner MF, Wolfer WG (1983) Numerical evaluation of pathintegral solutions to Fokker-Planck equations. 2. Restricted stochastic-processes. Phys Rev A 28(5):3003-3011

17. Wehner MF, Wolfer WG (1987) Numerical evaluation of pathintegral solutions to Fokker-Planck equations. 3. Time and functionally dependent coefficients. Phys Rev A 35(4):1795-1801

18. Naess A, Moe V (2000) Efficient path integration methods for nonlinear dynamic systems. Probab Eng Mech 15(2):221-231

19. Zorzano MP, Mais H, Vazquez L (1999) Numerical solution of two dimensional Fokker-Planck equations. Appl Math Comput 98(23):109-117

20. Kumar P, Narayanan S, Gupta S (2014) Finite element solution of Fokker-Planck equation of nonlinear oscillators subjected to colored non-gaussian noise. Probab Eng Mech 38:143-155

21. Fukushima H, Uesaka Y, Nakatani Y, Hayashi N (2002) Numerical solutions of the Fokker-Planck equation by the finite difference method for the thermally assisted reversal of the magnetization in a single-domain particle. J Magn Magn Mater 242:1002-1004

22. Kumar P, Narayanan S (2006) Solution of Fokker-Planck equation by finite element and finite difference methods for nonlinear systems. Sadhana-Acad Proc Eng Sci 31:445-461

23. Hsu CS (1981) A generalized theory of cell-to-cell mapping for non-linear dynamical-systems. J Appl Mech Trans ASME 48(3):634-642

24. Chen L, Sun JQ (2016) The closed-form steady-state probability density function of van der pol oscillator under random excitations. J Appl Nonlinear Dyn 5(4):495-502

25. Chen L, Sun JQ (2016) The closed-form solution of the reduced Fokker-Planck-Kolmogorov equation for nonlinear systems. Commun Nonlinear Sci Numer Simul 41:1-10 
26. Chen L, Liu J, Sun JQ (2017) Stationary response probability distribution of SDOF nonlinear stochastic systems. J Appl Mech 84(5):051006

27. Chen L, Qian J, Zhu H, Sun JQ (2019) The closed-form stationary probability distribution of the stochastically excited vibro-impact oscillators. J Sound Vib 439:260-270

28. Chen L, Sun JQ (2020) A highly-efficient method for stationary response of MDOF nonlinear stochastic systems. Appl Math Mech 41(6):967-982

29. Han J, Jentzen A, Weinan E (2018) Solving high-dimensional partial differential equations using deep learning. PNAS 115(34):8505-8510

30. Sirignano J, Spiliopoulos K (2018) DGM: A deep learning algorithm for solving partial differential equations. arXiv: $1708.07469 v 5$ [q-finMF]
31. Müller J, Zeinhofer M (2020) Deep Ritz revisited. arXiv: 1912.03937v2 [mathNA]

32. He J, Li L, Xu J, Zheng C (2018) ReLU deep neural networks and linear finite elements. arXiv:1807.03973v2 [mathNA]

33. Lagaris IE, Likas AC, Papageorgiou DG (2000) Neural-network methods for boundary value problems with irregular boundaries. IEEE Trans Neural Netw 11(5):1041-1049

34. Park J, Sandberg IW (1991) Universal approximation using radialbasis-function networks. Neural Comput 3:246-257

35. Wu Y, Wang H, Zhang B, Du KL (2012) Using radial basis function networks for function approximation and classification. ISRN Appl Math 2012:1-34. https://doi.org/10.5402/2012/324194

36. Nelles O (2001) Nonlinear system identification-from classical approaches to neural networks and fuzzy models. Springer, Berlin 